\title{
Comparative study of Apamarga Pratisaraniya Kshara and Palasha Pratisaraniya Kshara in the management of Ardra Arsha
}

\author{
Research Article
}

\section{Avnish Pathak ${ }^{*}$, Hemantha Kumar $\mathbf{P}^{2}$}

1. Ph.D scholar, 2. HOD Department of Shalya Tantra, SDM College of Ayurveda, Hassan, Karnataka

\begin{abstract}
To compare the effect of Apamarga Pratisaraniya Kshara and Palasha Pratisaraniya Kshara in the management of Ardra Arsha. Cases presenting with classical signs and symptoms of Ardra Arsha were selected. They were randomly allocated into two groups i.e. Group-AP and Group-PP. In Group-AP Apamarga Pratisaraniya Kshara was applied and in Group-PP Palasha Pratisaraniya Kshara was applied. The signs and symptoms of Ardra Arsha i.e. bleeding per rectum, Pain, Burning sensation, and size of Arshas were assessed before and after the completion of treatment. The relief provided by the therapy in pain, bleeding, discharge and reduction in size of the pile mass was highly significant $(\mathrm{P}<001)$. After the 15 days of the Kshara application 100\% relief in pain, bleeding, reduction in sloughing and total reduction in size of pile mass was recorded. On the basis of the comparison of the effects as mentioned above it can be said that the application of Apamarga Kshara provided better relief in pain, bleeding, slough and size of the pile mass of the patients in comparison to Palasha Kshara. Group-AP cases showed better improvement when compared to Group-PP. However all 15 cases of Group-AP and 15 cases of Group-PP showed good results.
\end{abstract}

Key Words: Ardra Arsha, Apamarga Pratisaraniya Kshara, Palasha Pratisaraniya Kshara, Mahanarayana Taila.

\section{Introducton}

Preservation and promotion of health as well as longevity have been a challenge in the history of mankind. Shalya Tantra, the integral part of Ayurvedic system contains detail description of Shastra Karma along with certain Para surgical procedures such as Kshara Karma, Agni Karma, and

*Corresponding Author:

\section{Avnish Pathak}

Ph.D scholar,

Department of Shalya Tantra,

SDM College of Ayurveda,

Hassan, Karnataka

Email: dravinishms@gmail.com

Ph. No: +91 - 9886593705
Jaloukavacharana. among these Para surgical measures, Kshara Karma is having supreme place due to its tremendous properties in curing diseases like Arsha and thus it can even replace Shastra Karma.. Apamarga and Palasha both drugs, are easily available, easy for collection so selected for the present study.

\section{Methodology}

The materials required for the procedures are - drugs, equipment, and patients with first and second degree haemorrhoids.

Drugs: The drug is Teekshna Palasha Kshara. 


\section{Preparation of Kshara}

$10 \mathrm{~kg}$ of whole plant of Palasha (Butea monosperma) was collected and was burnt into ash, and then it was allowed to cool by itself. The whole ash was collected $(1 \mathrm{~kg})$ and mixed with six liters of water and stirred well, allowed to settle overnight. Then it was filtered through double folded cloth for 21 times, residue was thrown out. Amber coloured filtrate was obtained. This was subjected to Mandagni. When the content was reduced to half, about $1 / 3$ of Kshara Jala was taken out of the vessel. $100 \mathrm{gm}$ of Sea shells ( Shukti) was heated red hot and then mixed with $1 / 3$ Kshara Jala to dissolve it completely. Thus dissolved Shukti is added to boiling Kshara Jala (Avapa stage) and continued to boil. Meanwhile $10 \mathrm{gm}$ of Citrakamoola (Plubago zeylanica) Kalka is added to the boiling Kshara Jala (prativapa stage) and allowed to boil for a few more minutes, till the content attained consistency as described by Susruta (not too liquid or too solid). Then it is removed from boiling and transferred into separate container with lid and stored for use. $\mathrm{P}_{\mathrm{H}}$ value of the Kshara obtained was $13.5\left(\mathrm{P}_{\mathrm{H}}\right.$ value of a strong alkali is 7 to 14).

The other drugs useful in the procedure of Kshara Karma are Xylocaine jelly, Lemon juice, and Mahanarayana taila.

Figure- 7 Palasha Kshara

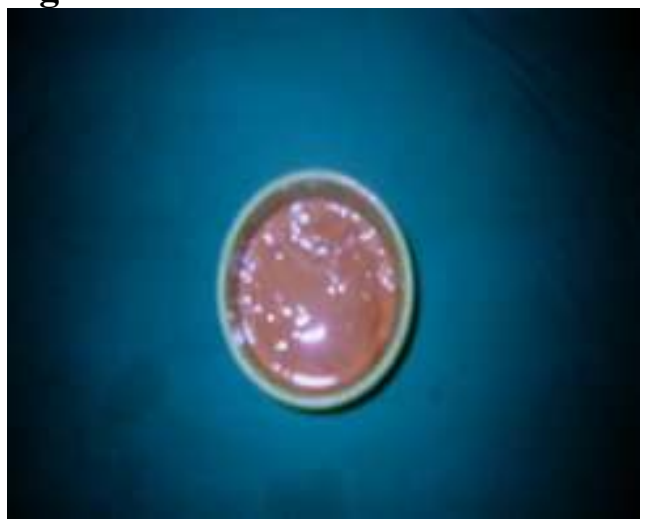

Figure -8 Apamarga Kshara

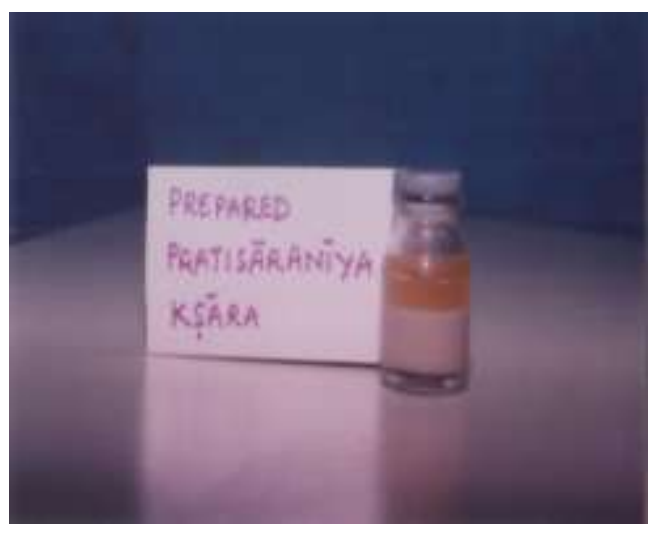

\section{Clinial study:}

30 patients were selected for the study from OPD and IPD of S.D.M. College of Ayurveda,Hassan Karnataka. The patients of different age, Sex and walks of life. They were divided into 2 groups. The criteria for the selection of the patient were as follows:

\section{Inclusion Criteria:}

- Patients above the age of 12 yrs.

- Patients suffering from Ardra Arsha having Pittaja and Rakthaja Lakshna.

- Arsha, which are of Mrudu, Prasrutha, Avagada (deep), Uchrita (projected).

- Second degree pile mass.

\section{Exclusion Criteria:}

- Patients bellow 12 yrs of age.

- Patients with Bahya Arsha.

- Patients contraindicated for Kshara Karma.

- Patients having other disorders like Diabetes mellitus, Malignancy, Chronic renal failure and patients with retroviral illness.

- Patients with complications like Gulma, Pleeha roga, Yakrut roga, Udara roga, Hasta Pada shotha, Hrit Parshwa Shoola and Ashteela.

Materials taken for the study: APgroup - Managed with Apamarga Kshara.

PPGroup - Managed with Palasha Kshara. 
Table No - 1 Showing the Grouping of the Patients

\begin{tabular}{|l|l|l|}
\hline Group & Treatment & Duration \\
\hline $\begin{array}{l}\text { AP } \\
\text { Group }\end{array}$ & $\begin{array}{l}\text { Apamarga } \\
\text { Pratisaraneeya } \\
\text { Kshara was } \\
\text { applied to the pile } \\
\text { mass. }\end{array}$ & $\begin{array}{l}\text { One sitting } \\
\text { and } \\
\text { observed for } \\
\text { 21 days. }\end{array}$ \\
\hline $\begin{array}{l}\text { PP } \\
\text { Group }\end{array}$ & $\begin{array}{l}\text { Palasha } \\
\text { Pratisaraneeya } \\
\text { Kshara was } \\
\text { applied to the pile } \\
\text { mass. }\end{array}$ & $\begin{array}{l}\text { One sitting } \\
\text { and } \\
\text { observed for } \\
\text { 21 days. }\end{array}$ \\
\hline
\end{tabular}

\section{Procedure of Kshara Karma:}

Pre-operative Procedures;

- Patient selected for the procedure were asked to remain nil orally for atleast 4 hours prior to the procedure.

- Part preparation was done

- Soap water enema was given

- Pre medications and anaesthesia were administered according to the need.

\section{Operative Procedure:}

Patient was made to lie down in lithotomic position; anus and surrounding area were cleaned with antiseptic lotion. Draping was done. Local anaesthesia with $2 \%$ xylocaine was administered. Manual anal dilatation was done sufficient enough to admit four fingers. Lubricated normal proctoscope was introduced, position of pile mass was noted and proctoscope was removed. Then slit proctoscope was introduced and skin around pile mass was pulled laterally with Alli's tissue holding forceps to get a better view of haemorrhoids.

The healthy anal mucosa was covered with wet cotton balls to prevent spilling of Kshara on it. Then the pile mass was gently scraped with the rough surface of spatula. Then Kshara was applied over pile mass, and the opening of proctoscope is closed for about 1 to $1 \frac{1}{2}$ minute Shata matra kala) with the palm. Then the pile mass was cleaned with lemon juice.

Observed that whether the pinkish pile mass was turned to blackish (Pakva Jambu Phala Varna). If not, Kshara was applied once again till the pile mass turned to blackish colour. Once again it was washed with lemon juice and sterile water wash was given. This procedure was repeated on all the pile masses. Thereafter the anal canal was packed with gauze piece soaked in Mahanarayana Taila to prevent pain, and local oedema. Dry dressing was done and the patient was shifted to ward.

\section{Post operative procedure:}

- Patient was kept nil by mouth for 6 hours after the Procedure.

- Packing was removed after 6 hours, and $5 \mathrm{ml}$ of Mahanarayana Taila was administered per rectally. From next day onwards patient was advised to take Panchavalkala Kvatha Sitz bath after passing motion for 10-15 mins twice a daily and Mahanarayana Taila is pushed rectaly after Sitz bath.

- Shatshakara churna or Triphala Choorna in dose of 1 tsf was given at night with Luke warm water as a laxative.

- Diet restriction was advised to the patient. Analgesics were administered according to the need. 


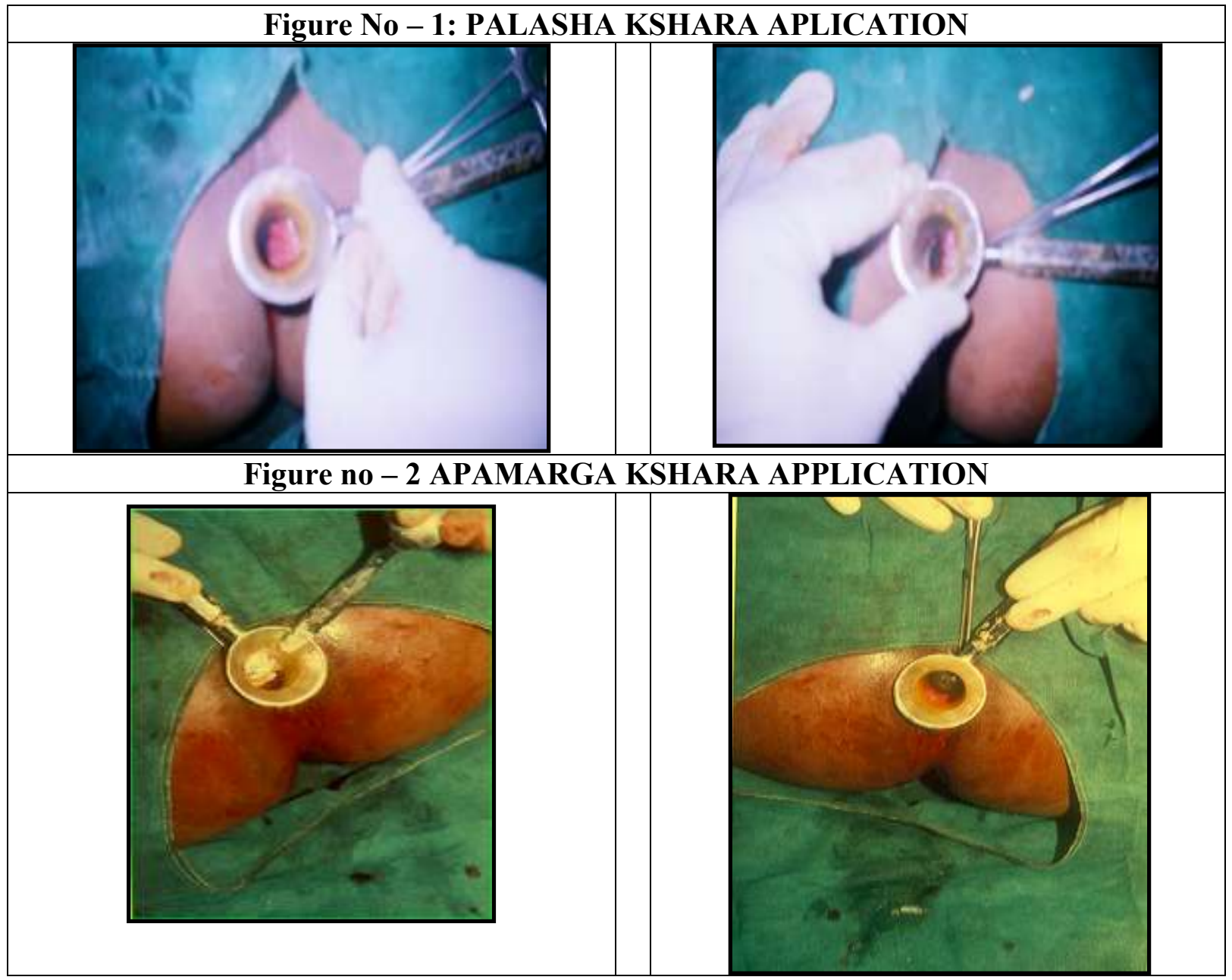

\section{Assessment Criteria}

- Ruja (Post operative pain)

- Raktha Srava (Bleeding)

- Size of Pile mass

- Post operative blackish brown discharge (Slough).

- Colour of Pile mass.

\section{Pain / Ruja:}

As the patient or the sufferer himself expresses the pain in his terms it was graded from mild to severe during defecation.

Table No - 2

\begin{tabular}{|l|l|}
\hline Symptoms & Grading \\
\hline No pain & 0 \\
\hline Pain during defecation & 1 \\
\hline Pain reduces within 30 mins & 2 \\
\hline
\end{tabular}

\begin{tabular}{|l|l|}
\hline after defecation & \\
\hline Pain during rest also & 3 \\
\hline
\end{tabular}

\section{Bleeding per Rectum / Rakta Srava:}

The grading was made based on patient's version. Attempts were made to have a gross quantitative analysis of bleeding in terms of mild, moderate and profuse. The grading is listed below:

Table No - 3

\begin{tabular}{|l|l|}
\hline Symptoms & Grading \\
\hline No bleeding & 0 \\
\hline Bleeding only during defecation & 1 \\
\hline $\begin{array}{l}\text { Bleeding during and after } \\
\text { defecation which lasts for 30 } \\
\text { mins or below }\end{array}$ & 2 \\
\hline $\begin{array}{l}\text { Bleeding irrespective to } \\
\text { defecation process }\end{array}$ & 3 \\
\hline
\end{tabular}


Size of Haemorrhoidal Mass / Arshankura:

This objective criteria is assessed with the help of proctoscopic examination. After operative procedure, oedema and slough were observed in both the groups, and these two are also given gradings which are enlisted below in tables. Proctoscopic examination was done daily upto 7 days to note the gradings.

Table No - 4

\begin{tabular}{|l|l|}
\hline Symptoms & Grading \\
\hline $\begin{array}{l}\text { Complete reduction of pile } \\
\text { mass }\end{array}$ & 0 \\
\hline $\begin{array}{l}\text { Moderate reduction in size of } \\
\text { pile mass }\end{array}$ & 1 \\
\hline $\begin{array}{l}\text { Slight reduction in size of pile } \\
\text { mass }\end{array}$ & 2 \\
\hline $\begin{array}{l}\text { No change in size of the pile } \\
\text { mass }\end{array}$ & 3 \\
\hline
\end{tabular}

Table No - 5 Srava / Post-Operative Discharge (Slough):

\begin{tabular}{|l|l|}
\hline Symptoms & Grading \\
\hline No slough & 0 \\
\hline $\begin{array}{l}\text { Slight slough, where examiner } \\
\text { observes during proctoscopic } \\
\text { examination }\end{array}$ & 1 \\
\hline $\begin{array}{l}\text { Patient notices slough on his } \\
\text { pads or undergarments }\end{array}$ & 2 \\
\hline
\end{tabular}

Excessive patient changes pads 3 or under garments due to wetting

Table No - 6 Colour of the Pile mass.

\begin{tabular}{|l|c|}
\hline Pakva Jambu Phala Varna & 3 \\
\hline Slightly blackish colour & 2 \\
\hline Slight pinkish colour & 1 \\
\hline Pinkish red colour & 0 \\
\hline
\end{tabular}

\section{Follow up study:}

After healing of Arsha lesion the Patients were asked to report at an interval of one month to the Shalya Tantra OPD to know whether the recurrence of Arsha occurred. The follow up study was continued for six months.

\section{Observations and Results}

In the present study, 30 patients suffering from Ardra Arsha fulfilling the inclusion criteria were studied. Patients were randomly categorized into Group-AP (Apamarga Pratisaraneeya Kshara) and Group-PP (Palasha Pratisaraneeya Kshara. There were no drop outs in this study. Following pages describes statistical analysis of the patients along with the observations and results:

Table-7. Age Wise Distribution of 30 Patients of Ardra Arsha

\begin{tabular}{|l|l|l|l|l|l|l|}
\hline \multirow{2}{*}{$\begin{array}{l}\text { Age } \\
\text { (in years) }\end{array}$} & Group-AP & Group-PP & Total \\
\cline { 2 - 7 } & No. & \% & No. & \% & No. & $\%$ \\
\hline $15-25$ & 04 & 27 & 00 & 00 & 04 & 13 \\
\hline $26-35$ & 08 & 53 & 07 & 47 & 15 & 50 \\
\hline $36-45$ & 02 & 13 & 04 & 27 & 06 & 20 \\
\hline $46-55$ & 01 & 07 & 04 & 27 & 05 & 17 \\
\hline
\end{tabular}

Age Groups: In this series of 30 patients of Ardra Arsha maximum number of the patients $(50 \%)$ was from the age group of $26-35$ years. It followed by $20 \%$ patients belonging to $36-$ 45 years, $17 \%$ to $46-55$ years and minimum i.e. $13 \%$ to $15-25$ years age groups (Table-11).

Table-8. Sex Wise Distribution of Patients

\begin{tabular}{|l|l|l|l|l|l|l|}
\hline \multirow{2}{*}{ Sex } & \multicolumn{4}{l}{ Group-AP } & Group-PP & Total \\
\cline { 2 - 7 } & No. & \% & No. & \% & No. & \% \\
\hline Male & 08 & 47 & 08 & 47 & 16 & 53 \\
\hline
\end{tabular}


Female

07

53

07

53

14

Sex: In this series maximum patients i.e. $53 \%$ were of male sex and remaining $47 \%$ were females (Table-12)

Table-9. Distribution of 30 Patients According to Religion

\begin{tabular}{|l|l|l|l|l|l|l|}
\hline \multirow{2}{*}{ Religion } & \multicolumn{4}{|l|}{ Group-AP } & Group-PP & Total \\
\cline { 2 - 7 } & No. & \% & No. & \% & No. & \% \\
\hline Hindu & 12 & 80 & 09 & 60 & 21 & 70 \\
\hline Muslim & 02 & 13 & 04 & 27 & 06 & 20 \\
\hline Christian & 01 & 07 & 02 & 13 & 03 & 10 \\
\hline
\end{tabular}

Religion: In this series maximum of $70 \%$ patients were Hindus, $20 \%$ were Muslims and a minimum of $10 \%$ patients were Christians (Table-13).

Table-10. Distribution of 30 Patients According to Marital Status

\begin{tabular}{|l|l|l|l|l|l|l|}
\hline \multirow{2}{*}{ Marital Status } & \multicolumn{4}{l}{ Group-AP } & Group-PP & Total \\
\cline { 2 - 7 } & No. & \% & No. & \% & No. & $\%$ \\
\hline Unmarried & 02 & 13 & 01 & 07 & 03 & 10 \\
\hline Married & 13 & 87 & 14 & 93 & 27 & 90 \\
\hline
\end{tabular}

Marital Status: This series consist maximum of $90 \%$ married patients and a minimum $10 \%$ of unmarried patients (Table-14).

Table-11. Distribution of 30 Patients According to the Occupation

\begin{tabular}{|l|l|l|l|l|l|l|}
\hline \multirow{2}{*}{ Occupation } & \multicolumn{4}{l|}{ Group-AP } & Group-PP & \multicolumn{2}{l|}{ Total } \\
\cline { 2 - 7 } & No. & \% & No. & \% & No. & $\%$ \\
\hline Sedentary & 6 & $40 \%$ & 7 & $47 \%$ & 13 & $43 \%$ \\
\hline Manual & 6 & $40 \%$ & 7 & $47 \%$ & 13 & $43 \%$ \\
\hline Labour & 3 & $20 \%$ & 1 & $7 \%$ & 4 & $13 \%$ \\
\hline
\end{tabular}

Occupation: In this series maximum of $43 \%$ patients were noted with both Sedentary and Manual occupations whereas 13\% were laborers by occupation (Table-15).

Table-12. Distribution of 30 Patients According to Socio-Economic Status

\begin{tabular}{|l|l|l|l|l|l|l|}
\hline \multirow{2}{*}{$\begin{array}{l}\text { Socio-Economic } \\
\text { Status }\end{array}$} & Group-AP & Group-PP & Total \\
\cline { 2 - 7 } & No. & \% & No. & \% & No. & $\%$ \\
\hline Poor & 08 & 53 & 06 & 40 & 14 & 47 \\
\hline Lower Middle & 02 & 13 & 02 & 13 & 04 & 13 \\
\hline Middle & 01 & 07 & 04 & 27 & 05 & 17 \\
\hline Upper Middle & 03 & 20 & 03 & 20 & 06 & 20 \\
\hline Rich & 01 & 07 & 00 & 00 & 01 & 03 \\
\hline
\end{tabular}

Socio-Economic Status: The maximum patients of this series i.e. $47 \%$ came from poor class of the society, $20 \%$ came from upper middle class, $17 \%$ from middle class, $13 \%$ came from lower middle class and a minimum of $3 \%$ were rich (Table-16).

Table-13. Signs and Symptoms Noted in 30 Patients of Arsha

\begin{tabular}{|c|c|c|c|c|c|c|}
\hline \multirow{2}{*}{ Signs/Symptoms } & \multicolumn{2}{|c|}{ Group-AP } & \multicolumn{2}{|c|}{ Group-PP } & \multicolumn{2}{|c|}{ Total } \\
\hline & No. & $\%$ & No. & $\%$ & No. & $\%$ \\
\hline $\begin{array}{ll}\text { Raktayukta } & \text { mala } \\
\text { Pravruthi } & \end{array}$ & 10 & 67 & 09 & 60 & 19 & 63 \\
\hline Daha & 03 & 20 & 03 & 20 & 06 & 20 \\
\hline
\end{tabular}


Ruja 02

13

03

20

05

17

Classical Signs and Symptoms of Arsha: In this series maximum of $63 \%$ patients complained of Raktayuktha Mala Pravruthi, 20\% were having Daha and 17\% of them were having Ruja (Table-17).

Table-14. Severity of Raktayukta Mala Pravrutti in 30 Patients of Ardra Arsha

\begin{tabular}{|l|l|l|l|l|l|l|}
\hline \multirow{2}{*}{ Severity } & \multicolumn{4}{|l|}{ Group-AP } & Group-PP & Total \\
\cline { 2 - 7 } & No. & \% & No. & \% & No. & \% \\
\hline Absent & 2 & 7 & 1 & 7 & 3 & 10 \\
\hline Mild & 4 & 27 & 3 & 20 & 7 & 24 \\
\hline Moderate & 5 & 33 & 5 & 33 & 10 & 33 \\
\hline Severe & 4 & 27 & 6 & 40 & 10 & 33 \\
\hline
\end{tabular}

Severity of Raktayukta Mala Pravrutti: In this series a maximum of 33\% patients each were having Moderate to Severe Rakthayukta Mala Pravrutti, 24\% of Patients had the mild symptom and a minimum of $10 \%$ had no such symptom (Table-18).

Table-15. Distribution of the Patients According to Severity of Daha

\begin{tabular}{|l|l|l|l|l|l|l|}
\hline \multirow{2}{*}{ Severity } & Group-AP & Group-PP & Total \\
\cline { 2 - 7 } & No. & \% & No. & \% & No. & $\%$ \\
\hline Absent & 12 & 80 & 11 & 73 & 23 & 77 \\
\hline Mild & 1 & 7 & 2 & 13 & 3 & 10 \\
\hline Moderate & 2 & 13 & 2 & 13 & 4 & 13 \\
\hline Severe & 0 & 0 & 0 & 0 & 0 & 0 \\
\hline
\end{tabular}

Daha according to Severity: In this Series only $23 \%$ were having Daha. Out of which 13\% of the patients were having moderate Daha and 10\% were having mild Daha and none was having Severe Daha (Table-19).

Table-16. Distribution of 30 Patients According to Severity of Ruja

\begin{tabular}{|l|l|l|l|l|l|l|}
\hline \multirow{2}{*}{ Severity of Ruja } & Group-AP & Group-PP & Total \\
\cline { 2 - 7 } & No. & \% & No. & \% & No. & \% \\
\hline Absent & 13 & 87 & 12 & 80 & 25 & 83 \\
\hline Mild & 1 & 7 & 2 & 13 & 3 & 10 \\
\hline Moderate & 1 & 7 & 1 & 7 & 2 & 7 \\
\hline Severe & 0 & 0 & 0 & 0 & 0 & 0 \\
\hline
\end{tabular}

Ruja: In this Series maximum of $83 \%$ patients were not having Ruja. $10 \%$ of the patients' complaint of mild Ruja and 7\% of them had moderate Ruja and none had Severe Ruja (Table20).

Table-17. Distribution of 30 patients of Ardra Arsha According to Chronicity

\begin{tabular}{|l|l|l|l|l|l|l|}
\hline \multirow{2}{*}{ Chronicity } & Group-AP & \multicolumn{2}{l|}{ Group-PP } & Total \\
\cline { 2 - 7 } & No. & \% & No. & \% & No. & $\%$ \\
\hline$<6$ months & 1 & 7 & 2 & 13 & 3 & 10 \\
\hline 6 months-1yr. & 3 & 20 & 3 & 20 & 6 & 20 \\
\hline 1yr.-2yr. & 4 & 27 & 3 & 20 & 7 & 23 \\
\hline 2 yr.-3yr. & 5 & 33 & 6 & 40 & 11 & 37 \\
\hline$>3$ yrs. & 2 & 13 & 1 & 7 & 3 & 10 \\
\hline
\end{tabular}


Chronicity: In this series, maximum of $37 \%$ patients had less than 6 months chronicity, followed by $23 \%$ having chronicity of 6 months- 1 years, $20 \%$ had $1-2$ years chronicity and $10 \%$ each had 2-3years and more than 3years each (Table-21).

Table-18. 30 Patients of Ardra Arsha According to Mode of Onset

\begin{tabular}{|c|c|c|c|c|c|c|}
\hline \multirow{2}{*}{ Mode of Onset } & \multicolumn{2}{|c|}{ Group-AP } & \multicolumn{2}{|c|}{ Group-PP } & \multicolumn{2}{|c|}{ Total } \\
\hline & No. & $\%$ & No. & $\%$ & No. & $\%$ \\
\hline Acute & 4 & 27 & 6 & 40 & 10 & 33 \\
\hline Gradual & 11 & 73 & 9 & 60 & 20 & 67 \\
\hline
\end{tabular}

Mode of Onset: In this series maximum of $67 \%$ patients had acute onset followed by $33 \%$ of them having gradual onset (Table-22).

Table-19. 30 of Patients of Ardra Arsha According to the Family History

\begin{tabular}{|l|l|l|l|l|l|l|}
\hline \multirow{2}{*}{ Family History } & \multicolumn{3}{|l|}{ Group-AP } & \multicolumn{2}{l|}{ Group-PP } & Total \\
\cline { 2 - 7 } & No. & \% & No. & \% & No. & $\%$ \\
\hline Present & 10 & 67 & 11 & 73 & 21 & 70 \\
\hline Absent & 5 & 33 & 4 & 27 & 9 & 30 \\
\hline
\end{tabular}

Family History: In this series, a maximum of $70 \%$ patients had positive familial history of Arsha and remaining $30 \%$ patients were not having the familial history of this disease (Table23).

Table-20. Distribution of 30 Patients According to the Addiction

\begin{tabular}{|l|l|l|l|l|l|l|}
\hline \multirow{2}{*}{ Habits } & Group-AP & Group-PP & Total \\
\cline { 2 - 7 } & No. & \% & No. & \% & No. & $\%$ \\
\hline Smoking & 5 & 33 & 6 & 40 & 11 & 37 \\
\hline Alcohol & 6 & 40 & 4 & 27 & 10 & 33 \\
\hline Tobacco chewing & 0 & 0 & 2 & 13 & 2 & 7 \\
\hline No Habits & 4 & 27 & 3 & 20 & 7 & 23 \\
\hline
\end{tabular}

Addiction: In this series 37\% Patients had the habit of smoking followed by $33 \%$ were having habit of alcohol intake and $7 \%$ had the habit of tobacco chewing (Table-24).

Table-21. Distribution of 30 Patients According to the Diet Habit

\begin{tabular}{|c|c|c|c|c|c|c|}
\hline \multirow{2}{*}{ Type of Ahara } & \multicolumn{2}{|c|}{ Group-AP } & \multicolumn{2}{|c|}{ Group-PP } & \multicolumn{2}{|c|}{ Total } \\
\hline & No. & $\%$ & No. & $\%$ & No. & $\%$ \\
\hline Vegetarian & 4 & 27 & 5 & 33 & 9 & 30 \\
\hline Mixed & 11 & 73 & 10 & 67 & 21 & 70 \\
\hline
\end{tabular}

Diet Habit: In this series $70 \%$ patients were taking mixed diet while remaining $30 \%$ patients were vegetarians (Table-25)

Table-22. 30 Patients of Ardra Arsha According to the Nidana Factors

\begin{tabular}{|l|l|l|l|l|l|l|}
\hline \multirow{2}{*}{ Nidana } & Group-AP & Group-PP & Total & \\
\cline { 2 - 7 } & No. & \% & No. & \% & No. & \% \\
\hline Viruddahara & 2 & 13 & 5 & 33 & 7 & 23 \\
\hline Vyavaya & 0 & 0 & 1 & 7 & 1 & 3 \\
\hline Adyashana & 1 & 7 & 3 & 20 & 4 & 13 \\
\hline Utkatukasana & 3 & 20 & 3 & 20 & 6 & 20 \\
\hline Prushtayana & 1 & 7 & 2 & 13 & 3 & 10 \\
\hline Vega Vidharana & 8 & 53 & 1 & 7 & 9 & 30 \\
\hline
\end{tabular}


Nidana Factors: In this series maximum of 30\% patients were having the habit of Vega Vidharana, followed by $23 \%$ patients were taking Virudhahara, $20 \%$ sitting in Uthkatakasana,13\% had Adhyashana, 10\% had Prushtayana and 3\% patients were indulged in Ati Vyavaya (Table-26).

Table-23. 30 Patients of Ardra Arsha According to the position of Pile Mass

\begin{tabular}{|l|l|l|l|l|l|l|}
\hline \multirow{2}{*}{ Position } & \multicolumn{4}{l}{ Group-AP } & Group-PP & Total \\
\cline { 2 - 7 } & No. & \% & No. & \% & No. & $\%$ \\
\hline 3' O Clock & 4 & 27 & 5 & 33 & 9 & 30 \\
\hline 7' O Clock & 9 & 60 & 7 & 47 & 16 & 53 \\
\hline $11 '$ ' Clock & 2 & 13 & 3 & 20 & 5 & 17 \\
\hline
\end{tabular}

Position of Pile Mass: In this series a maximum of 53\% patients had pile mass at 7' 0 Clock position followed by $30 \%$ had pile mass at 3 ' 0 clock position and $17 \%$ of them had pile mass at $11^{\prime} 0$ clock position (Table-27).

Table-24 Effect of Apamarga Pratisaraniya Kshara on Ruja

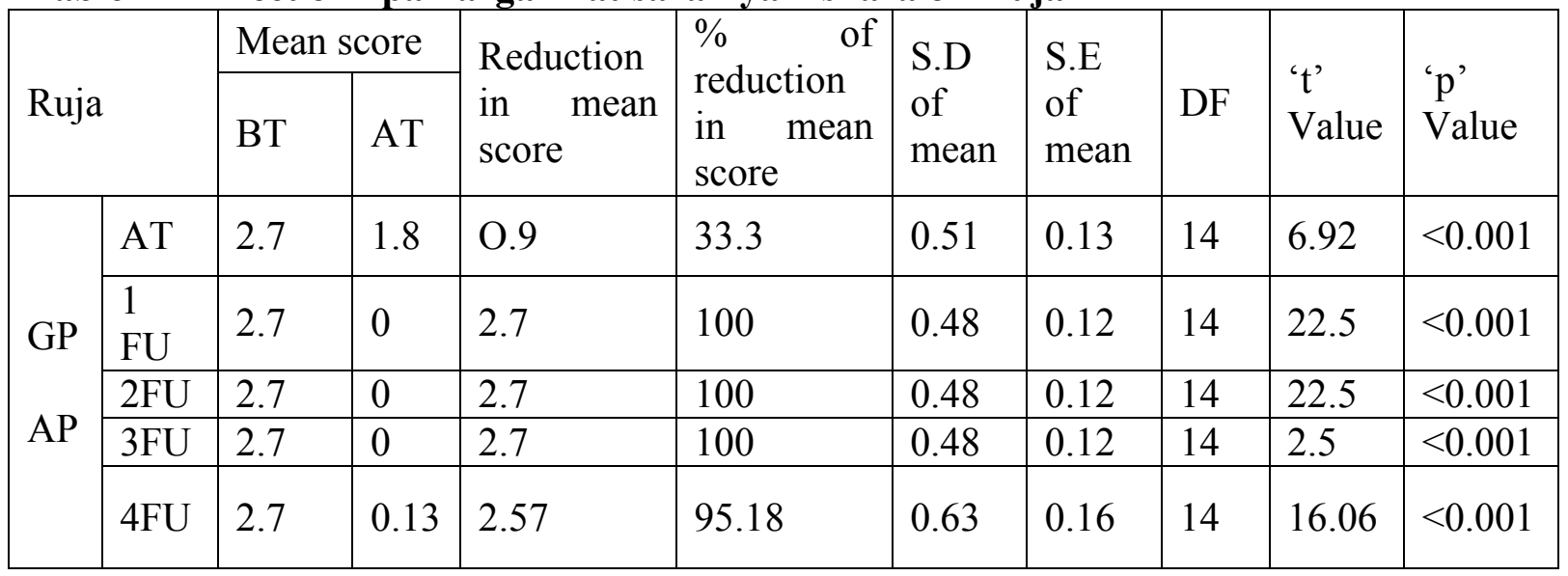

BT - Before Treatment, GP- group, AP- Apamarga Kshara, AT-After Treatment, SDStandard Deviation, SE- Standard Error, DF-degrees of freedom , FU- Follow up

Table-25 Effect of Apamarga Pratisaraniya Kshara on Rakta Srava

\begin{tabular}{|c|c|c|c|c|c|c|c|c|c|}
\hline \multirow{2}{*}{\multicolumn{2}{|c|}{ Raktha Srava }} & \multicolumn{2}{|c|}{ Mean score } & \multirow{2}{*}{$\begin{array}{l}\% \text { of } \\
\text { reduction in } \\
\text { mean score }\end{array}$} & \multirow{2}{*}{$\begin{array}{l}\text { S.D of } \\
\text { mean }\end{array}$} & \multirow{2}{*}{$\begin{array}{l}\text { S.E of } \\
\text { mean }\end{array}$} & \multirow{2}{*}{ Df } & \multirow{2}{*}{$\begin{array}{l}\text { ' } \mathrm{t} \text { ' } \\
\text { Value }\end{array}$} & \multirow{2}{*}{$\begin{array}{l}\text { 'p' } \\
\text { Value }\end{array}$} \\
\hline & & BT & AT & & & & & & \\
\hline \multirow{5}{*}{$\begin{array}{l}\text { Gp } \\
\text { AP }\end{array}$} & AT & 2.7 & 1.8 & 33.3 & 0.51 & 0.13 & 14 & 6 & $<0.001$ \\
\hline & $1 \mathrm{FU}$ & 2.7 & 0 & 100 & 0.48 & 0.12 & 14 & 22.5 & $<0.001$ \\
\hline & $2 \mathrm{FU}$ & 2.7 & 0 & 100 & 0.48 & 0.12 & 14 & 22.5 & $<0.001$ \\
\hline & $3 \mathrm{FU}$ & 2.7 & 0 & 100 & 0.48 & 0.12 & 14 & 22.5 & $<0.001$ \\
\hline & $4 \mathrm{FU}$ & 2.7 & 0.23 & 94.8 & 0.63 & 0.16 & 14 & 14.81 & $<0.001$ \\
\hline
\end{tabular}

BT - Before Treatment, GP- group, AP- Apamarga Kshara, AT-After Treatment, SDStandard Deviation, SE- Standard Error, DF-degrees of freedom , FU- Follow up 
Table-26 Effect of Apamarga Pratisaraniya Kshara on Srava (Slough)

\begin{tabular}{|c|c|c|c|c|c|c|c|c|c|}
\hline \multirow{2}{*}{\multicolumn{2}{|c|}{$\begin{array}{l}\text { Srava( } \\
\text { Slough) }\end{array}$}} & \multicolumn{2}{|c|}{$\begin{array}{l}\text { Mean } \\
\text { score }\end{array}$} & \multirow[t]{2}{*}{$\begin{array}{l}\% \text { of reduction in } \\
\text { mean score }\end{array}$} & \multirow[t]{2}{*}{$\begin{array}{l}\text { S.D of } \\
\text { mean }\end{array}$} & \multirow[t]{2}{*}{$\begin{array}{l}\text { S.E of } \\
\text { mean }\end{array}$} & \multirow[t]{2}{*}{ Df } & \multirow[t]{2}{*}{$\begin{array}{l}\text { ' } \mathrm{t} \text { ' } \\
\text { Value }\end{array}$} & \multirow[t]{2}{*}{$\begin{array}{l}\text { 'p' } \\
\text { Value }\end{array}$} \\
\hline & & $\mathrm{BT}$ & AT & & & & & & \\
\hline \multirow{5}{*}{$\begin{array}{l}\text { Gp } \\
\text { AP }\end{array}$} & AT & 2.7 & 1.5 & 44.4 & 0.74 & 0.19 & 14 & 6.31 & $<0.001$ \\
\hline & $\begin{array}{c}1 \\
\text { FU }\end{array}$ & 2.7 & 0 & 100 & 0.48 & 0.12 & 14 & 22.5 & $<0.001$ \\
\hline & $\begin{array}{c}2 \\
F U\end{array}$ & 2.7 & 0 & 100 & 0.48 & 0.12 & 14 & 22.5 & $<0.001$ \\
\hline & $\begin{array}{c}3 \\
\mathrm{FU}\end{array}$ & 2.7 & 0 & 100 & 0.48 & 0.12 & 14 & 22.5 & $<0.001$ \\
\hline & $\begin{array}{c}4 \\
\mathrm{FU}\end{array}$ & 2.7 & 0.13 & 100 & 0.74 & 0.19 & 14 & 14.2 & $<0.001$ \\
\hline
\end{tabular}

BT - Before Treatment, GP- group, AP- Apamarga Kshara, AT-After Treatment, SDStandard Deviation, SE- Standard Error, DF-degrees of freedom , FU- Follow up

Table-27 Effect of Apamarga Pratisaraniya Kshara on Size of Pile mass

\begin{tabular}{|c|c|c|c|c|c|c|c|c|c|}
\hline \multirow{2}{*}{\multicolumn{2}{|c|}{$\begin{array}{l}\text { Size of Pile } \\
\text { Mass }\end{array}$}} & \multicolumn{2}{|c|}{$\begin{array}{l}\text { Mean } \\
\text { score }\end{array}$} & \multirow[t]{2}{*}{$\begin{array}{l}\% \text { of reduction in } \\
\text { mean score }\end{array}$} & \multirow[t]{2}{*}{$\begin{array}{l}\text { S.D of } \\
\text { mean }\end{array}$} & \multirow[t]{2}{*}{$\begin{array}{l}\text { S.E of } \\
\text { mean }\end{array}$} & \multirow[t]{2}{*}{ Df } & \multirow[t]{2}{*}{$\begin{array}{l}\text { ' } t \text { ' } \\
\text { Value }\end{array}$} & \multirow[t]{2}{*}{$\begin{array}{l}\text { 'p' } \\
\text { Value }\end{array}$} \\
\hline & & BT & $\mathrm{AT}$ & & & & & & \\
\hline \multirow{5}{*}{$\begin{array}{l}\text { Gp } \\
\text { AP }\end{array}$} & AT & 2.7 & 1.3 & 51.9 & 0.72 & 0.18 & 14 & 7.77 & $<0.001$ \\
\hline & $1 \mathrm{FU}$ & 2.7 & 0 & 100 & 0.48 & 0.12 & 14 & 22.5 & $<0.001$ \\
\hline & $2 \mathrm{FU}$ & 2.7 & 0 & 100 & 0.48 & 0.12 & 14 & 22.5 & $<0.001$ \\
\hline & $3 \mathrm{FU}$ & 2.7 & 0 & 100 & 0.48 & 0.12 & 14 & 22.5 & $<0.001$ \\
\hline & $4 \mathrm{FU}$ & 2.7 & 0.13 & 100 & 0.63 & 0.16 & 14 & 22.5 & $<0.001$ \\
\hline
\end{tabular}

BT - Before Treatment, GP- group, AP- Apamarga Kshara, AT-After Treatment, SDStandard Deviation, SE- Standard Error, DF-degrees of freedom , FU- Follow up

Table-28 Effect of Apamarga Pratisaraniya Kshara on Colour of Mass

\begin{tabular}{|c|c|c|c|c|c|c|c|c|c|}
\hline \multirow{2}{*}{\multicolumn{2}{|c|}{$\begin{array}{l}\text { Srava( } \\
\text { Slough) }\end{array}$}} & \multicolumn{2}{|c|}{$\begin{array}{l}\text { Mean } \\
\text { score }\end{array}$} & \multirow[t]{2}{*}{$\begin{array}{l}\% \text { of reduction in } \\
\text { mean score }\end{array}$} & \multirow[t]{2}{*}{$\begin{array}{l}\text { S.D of } \\
\text { mean }\end{array}$} & \multirow[t]{2}{*}{$\begin{array}{l}\text { S.E of } \\
\text { mean }\end{array}$} & \multirow[t]{2}{*}{ Df } & \multirow[t]{2}{*}{$\begin{array}{l}\text { ' } t \text { ' } \\
\text { Value }\end{array}$} & \multirow[t]{2}{*}{$\begin{array}{l}\text { 'p' } \\
\text { Value }\end{array}$} \\
\hline & & BT & $\mathrm{AT}$ & & & & & & \\
\hline \multirow{5}{*}{$\begin{array}{l}\text { Gp } \\
\text { AP }\end{array}$} & AT & 2.7 & 1.8 & 33.3 & 0.51 & 0.13 & 14 & 6.92 & $<0.001$ \\
\hline & $1 \mathrm{FU}$ & 2.7 & 0 & 100 & 0.48 & 0.12 & 14 & 22.5 & $<0.001$ \\
\hline & $2 \mathrm{FU}$ & 2.7 & 0 & 100 & 0.48 & 0.12 & 14 & 22.5 & $<0.001$ \\
\hline & $3 \mathrm{FU}$ & 2.7 & 0 & 100 & 0.48 & 0.12 & 14 & 22.5 & $<0.001$ \\
\hline & $4 \mathrm{FU}$ & 2.7 & 0 & 100 & 0.63 & 0.16 & 14 & 22.5 & $<0.001$ \\
\hline
\end{tabular}

BT - Before Treatment, GP- group, AP- Apamarga Kshara, AT-After Treatment, SDStandard Deviation, SE- Standard Error, DF-degrees of freedom , FU- Follow up 
Table-29 Effects of Palasha Pratisaraniya Kshara on Ardra Arsha -Ruja

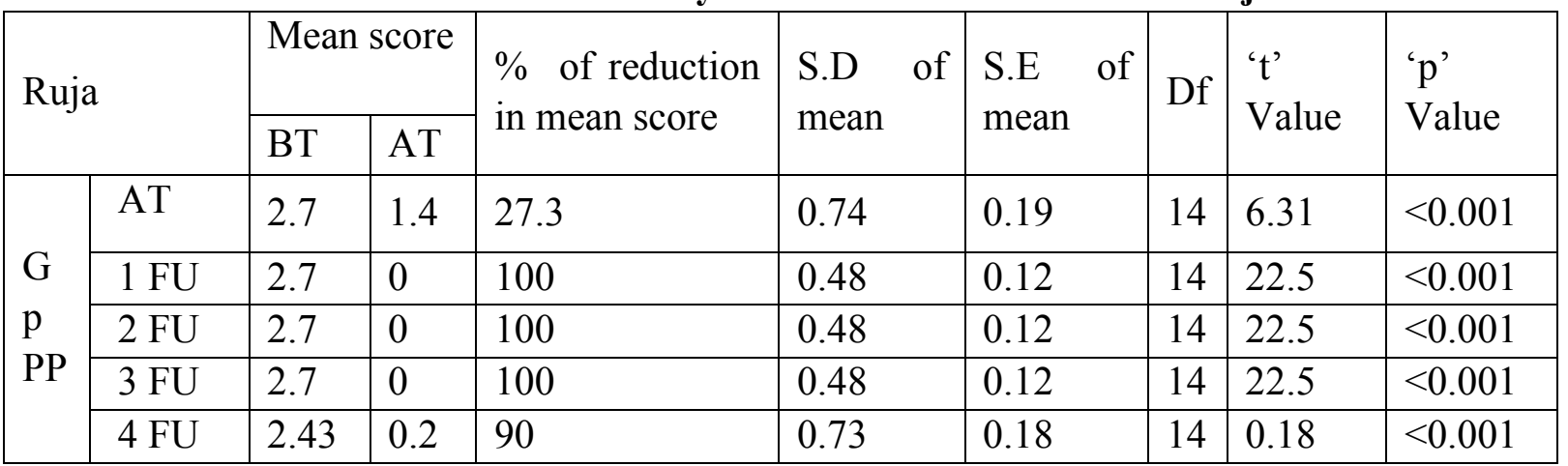

BT - Before Treatment, GP- group, AP- Apamarga Kshara, AT-After Treatment, SDStandard Deviation, SE- Standard Error, DF-degrees of freedom , FU- Follow up

Table-30 Effects of Palasha Pratisaraniya Kshara on the Patients of Ardra Arsha Raktha Srava

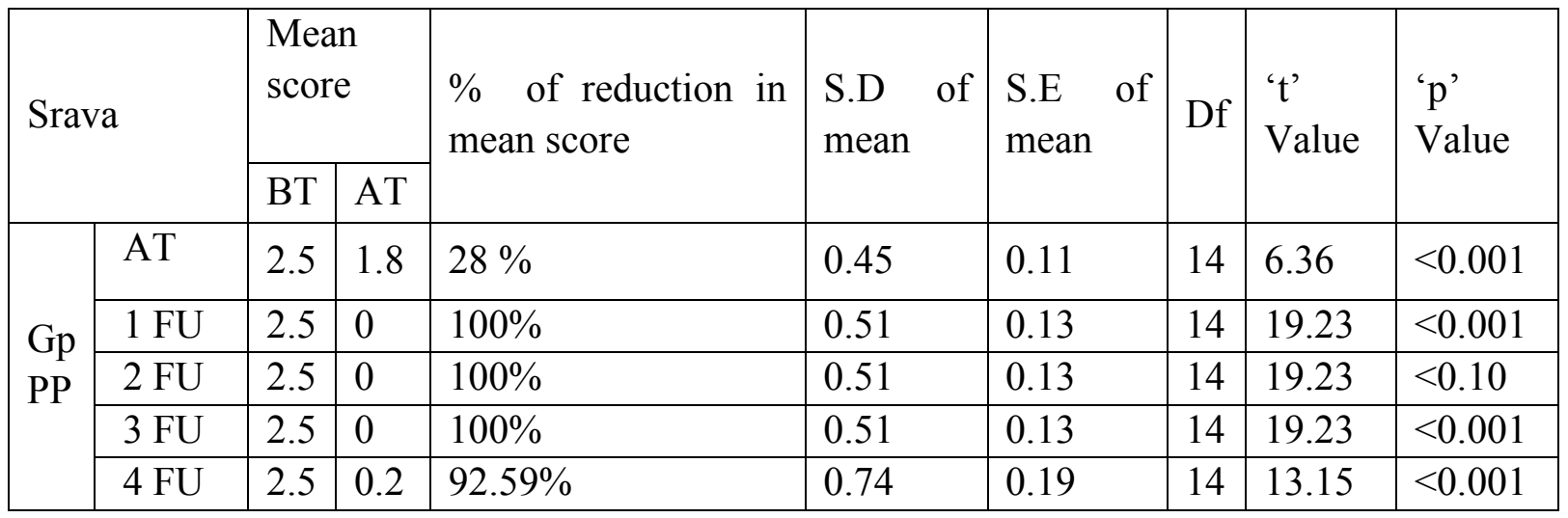

BT - Before Treatment, GP- group, PP- Palaksha Kshara, AT-After Treatment, SD- Standard

Deviation, SE- Standard Error, DF-degrees of freedom , FU- Follow up

Table-31 Effects of Palasha Pratisaraniya Kshara on the Patients of Ardra Arsha Srava (Discharge of Slough)

\begin{tabular}{|c|c|c|c|c|c|c|c|c|c|}
\hline \multirow{3}{*}{\multicolumn{2}{|c|}{$\begin{array}{l}\text { Srava( } \\
\text { Discharge of } \\
\text { slough) }\end{array}$}} & \multirow{2}{*}{\multicolumn{2}{|c|}{$\begin{array}{l}\text { Mean } \\
\text { score }\end{array}$}} & \multirow{3}{*}{$\begin{array}{l}\% \text { of reduction } \\
\text { in mean score }\end{array}$} & \multirow{3}{*}{$\begin{array}{l}\text { S.D of } \\
\text { mean }\end{array}$} & \multirow{3}{*}{$\begin{array}{l}\text { S.E of } \\
\text { mean }\end{array}$} & \multirow{3}{*}{ Df } & \multirow{3}{*}{$\begin{array}{l}\text { ' } \mathrm{t} \text { ' } \\
\text { Value }\end{array}$} & \multirow{3}{*}{$\begin{array}{l}\text { 'p' } \\
\text { Value }\end{array}$} \\
\hline & & & & & & & & & \\
\hline & & BT & $\mathrm{AT}$ & & & & & & \\
\hline \multirow{5}{*}{$\begin{array}{l}\text { Gp } \\
\text { PP }\end{array}$} & AT & 2.7 & 1.8 & $27.3 \%$ & 0.51 & 0.13 & 14 & 6.12 & $<0.001$ \\
\hline & $1 \mathrm{FU}$ & 2.7 & 0 & $100 \%$ & 0.48 & 0.12 & 14 & 22.5 & $<0.001$ \\
\hline & $2 \mathrm{FU}$ & 2.7 & 0 & $100 \%$ & 0.48 & 0.12 & 14 & 22.5 & $<0.001$ \\
\hline & $3 \mathrm{FU}$ & 2.7 & 0 & $100 \%$ & 0.48 & 0.12 & 14 & 22.5 & $<0.001$ \\
\hline & $4 \mathrm{FU}$ & 2.7 & 0 & $100 \%$ & 0.63 & 0.16 & 14 & 16.86 & $<0.001$ \\
\hline
\end{tabular}

BT - Before Treatment, GP- group, PP- Palaksha Kshara, AT-After Treatment, SD- Standard Deviation, SE- Standard Error, DF-degrees of freedom , FU- Follow up 
Table-32 Effects of Apamarga Pratisaraniya Kshara on the Patients of Ardra ArshaSize of pile mass

\begin{tabular}{|c|c|c|c|c|c|c|c|c|c|}
\hline \multirow{2}{*}{\multicolumn{2}{|c|}{$\begin{array}{l}\text { Size of pile } \\
\text { mass }\end{array}$}} & \multicolumn{2}{|c|}{ Mean score } & \multirow{2}{*}{$\begin{array}{l}\% \text { of } \\
\text { reduction in } \\
\text { mean score }\end{array}$} & \multirow{2}{*}{$\begin{array}{l}\text { S.D of } \\
\text { mean }\end{array}$} & \multirow{2}{*}{$\begin{array}{l}\text { S.E of } \\
\text { mean }\end{array}$} & \multirow{2}{*}{ Df } & \multirow{2}{*}{$\begin{array}{l}\text { ' } \mathrm{t} \text { ' } \\
\text { Value }\end{array}$} & \multirow{2}{*}{$\begin{array}{l}\text { 'p' } \\
\text { Value }\end{array}$} \\
\hline & & BT & AT & & & & & & \\
\hline \multirow{5}{*}{$\begin{array}{l}\text { Gp } \\
\text { PP }\end{array}$} & AT & 2.7 & 1.8 & $33.3 \%$ & 0.51 & 0.13 & 14 & 6.92 & $<0.001$ \\
\hline & $1 \mathrm{FU}$ & 2.7 & 0 & $100 \%$ & 0.48 & 0.12 & 14 & 22.5 & $<0.001$ \\
\hline & $2 \mathrm{FU}$ & 2.7 & 0 & $100 \%$ & 0.48 & 0.12 & 14 & 22.5 & $<0.10$ \\
\hline & $3 \mathrm{FU}$ & 2.7 & 0 & $100 \%$ & 0.48 & 0.12 & 14 & 22.5 & $<0.001$ \\
\hline & $4 \mathrm{FU}$ & 2.7 & 0.26 & $90.37 \%$ & 0.63 & 0.16 & 14 & 15.25 & $<0.001$ \\
\hline
\end{tabular}

BT - Before Treatment, GP- group, PP- Palaksha Kshara, AT-After Treatment, SD- Standard

Deviation, SE- Standard Error, DF-degrees of freedom , FU- Follow up

Table-33 Effects of Palasha Pratisaraniya Kshara on the Patients of Ardra ArshaColour of Pile mass.

\begin{tabular}{|c|c|c|c|c|c|c|c|c|c|}
\hline \multirow{2}{*}{\multicolumn{2}{|c|}{$\begin{array}{l}\text { Colour of pile } \\
\text { mass }\end{array}$}} & \multicolumn{2}{|c|}{ Mean score } & \multirow{2}{*}{$\begin{array}{ll}\% & \text { of } \\
\text { reduction in } \\
\text { mean score }\end{array}$} & \multirow{2}{*}{$\begin{array}{l}\text { S.D of } \\
\text { mean }\end{array}$} & \multirow{2}{*}{$\begin{array}{l}\text { S.E of } \\
\text { mean }\end{array}$} & \multirow{2}{*}{ Df } & \multirow{2}{*}{$\begin{array}{l}\text { ' } \mathrm{t} \text { ' } \\
\text { Value }\end{array}$} & \multirow{2}{*}{$\begin{array}{l}\text { 'p' } \\
\text { Value }\end{array}$} \\
\hline & & BT & $\mathrm{AT}$ & & & & & & \\
\hline \multirow{5}{*}{$\begin{array}{l}\text { Gp } \\
\text { PP }\end{array}$} & AT & 2.7 & 1.8 & $33.3 \%$ & 0.51 & 0.13 & 14 & 6.92 & $<0.001$ \\
\hline & $1 \mathrm{FU}$ & 2.7 & 0 & $100 \%$ & 0.48 & 0.12 & 14 & 22.5 & $<0.001$ \\
\hline & $2 \mathrm{FU}$ & 2.7 & 0 & $100 \%$ & 0.48 & 0.12 & 14 & 22.5 & $<0.001$ \\
\hline & $3 \mathrm{FU}$ & 2.7 & 0 & $100 \%$ & 0.48 & 0.12 & 14 & 22.5 & $<0.001$ \\
\hline & $4 \mathrm{FU}$ & 2.7 & 0 & $100 \%$ & 0.48 & 0.16 & 14 & 22.5 & $<0.001$ \\
\hline
\end{tabular}

BT - Before Treatment, GP- group, PP- Palaksha Kshara, AT-After Treatment, SD- Standard Deviation, SE- Standard Error, DF-degrees of freedom , FU- Follow up

Discussion

Table No: 34 Showing the Difference in Effect of Parameters in Both the Groups

\begin{tabular}{|l|l|l|l|l|l|}
\hline Sl.No & Ruja & Rakta- Srava & Srava(Slough) & $\begin{array}{l}\text { Size of Pile } \\
\text { Mass }\end{array}$ & $\begin{array}{l}\text { Colour } \\
\text { of Pile } \\
\text { Mass }\end{array}$ \\
\hline $\begin{array}{l}\text { Apamarga } \\
\text { Group }\end{array}$ & Reoccurrence & $\begin{array}{l}\text { No } \\
\text { Recurrence }\end{array}$ & $\begin{array}{l}\text { No } \\
\text { Recurrence }\end{array}$ & $\begin{array}{l}\text { No } \\
\text { Recurrence }\end{array}$ & No \\
\hline $\begin{array}{l}\text { Palasha } \\
\text { Group }\end{array}$ & Reoccurrence & Reoccurrence & $\begin{array}{l}\text { No } \\
\text { Recurrence }\end{array}$ & Reoccurrence & No \\
\hline
\end{tabular}

According to Ayurveda, Arsha is a Tridoshaja Vyadhi, though Raktaja Arsha finds a separate mention; it presents features of Pittaja Arsha with more bleeding tendency. The treatment modalities of haemorrhoids either medical, surgical or Para surgical, come under four treatment principles of Arsha told by Susruta. Even though Kshara Karma is a simple procedure, this is not being practiced by many, may be because of difficulty in preparing Pratisaraniya 
Kshara with correct potency. The criteria of assessing the correct potency of Pratisaraniya Kshara are getting Jambuphala Varna of red pile mass within Shatamatra Kala (approximately one minute). This has been achieved here by rigidly following the technique of Kshara preparation told by Susruta.

For the present study, drugs selected for Kshara preparation are Palasha, Apamarga, Sukti and Citraka. Apamarga is a freely available herb. Further, its usage in Arsha is recommended by most of Ayurveda Acharyas. Similarly, Palasha is also easily available and indicated in Arsha. Sukti is also available easily. Its usage in Kshara preparation is told by Susruta. $\mathrm{PH}$ value of alkali range from 7 to 14 . The $P_{H}$ value of Tikshna Apamarga Kshara prepared for the study was 13.5.

Histopathological examination of slough material in blackish brown discharge reveals the presence of necrosed haemorrhoidal tissue. This helps to draw the inference regarding probable mode of action of Pratisaraneeya Kshara.

\section{Probable Mode of Action}

Pratisaraneeya kshara acts on haemorrhoids in two ways -1 . It cauterizes the pile mass directly because of its Ksharana Guna (corrosive nature). 2. It coagulates protein in haemorrhoidal plexus.

The coagulation of protein leads to disintegration of haemoglobin into haem and globin. Synergy of these actions results in decreasing the size of the pile mass. Further, necrosis of the tissue in the haemorrhoidal vein will occur. This necrosed tissue slough out as blackish brown discharge for3 to 7 days. The haem present in the slough gives the discharge its colour. The tissue becomes fibrosed and scar formation seen. The haemorrhoidal vein obliterates permanently and there is no recurrence of haemorrhoids.

\section{Conclusion}

On the basis of the comparison of the effects of both the groups it can be said that the application of Apamarga Kshara provided better relief in pain, bleeding, slough and size of the pile mass of the patients in comparison to Palasha Kshara. Post operative slough was present for less number of days in Apamarga Pratisaraneeya Kshara when compared with Palasha Pratisaraneeya Kshara. The haemorrhoidal mass treated with Apamarga Kshara required minimum number of days when compared to Palasha Kshara to heal.

\section{References}

1. Kaviraja Ambikadatta Shastri, Sushruta Samhita,6th Edn, Hindi Comm reprint 2002Chaukhamba Sanskrit Samsthana, Varanasi, pp. 34

2. Kaviraja Ambikadatta Shastri, Sushruta (1987), Sushruta Samhita 6th Edn, Hindi Comm reprint 2002Chaukhamba Sanskrit

Samsthana, Varanasi, pp. 62-63

3. Chakrapani, Charaka Samhita, with Ayurveda Dipika Sanskrit Comm1991, Kaviraja Narendranath Sengupta and Balaichandra Sengupta Chaukhamba Orientalia, Varanasi, p. 786.

4. Arunadatta (1939), Astanga Hrudaya, 8th Edn, with Sarvanga Sundara, Sanskrit comm. Edr, Bhishagacharya Harisastri Paradakara vaidya, Chaukhamba Orientalia, Varanasi, pp. 225-228.

5. Sushruta (1987), Sushruta Samhita, 6th Edn, Hindi Comm, Edr, Kaviraja Ambikadatta Shastri, Chaukhamba Sanskrit Samsthana, Varanasi, pp. 35-36. 